\title{
The Fermi GBM and LAT follow-up of GW150914
}

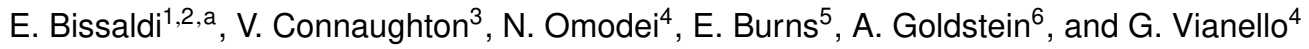 \\ ${ }^{1}$ Politecnico di Bari (Italy) \\ ${ }^{2}$ INFN - Sezione di Bari (Italy) \\ ${ }^{3}$ Universities Space Research Association, AL (USA) \\ ${ }^{4}$ W. W. Hansen Experimental Physics Laboratory, Kavli Institute for Particle Astrophysics and Cosmology, \\ Department of Physics and SLAC National Accelerator Laboratory, Stanford University, CA (USA) \\ ${ }^{5}$ Physics Department, University of Alabama in Huntsville, AL (USA) \\ ${ }^{6}$ NASA/Marshall Space Flight Center, AL (USA)
}

\begin{abstract}
As the first detection of Gravitation Wave (GW) event arising from the coalescence of two stellar-mass Black Holes (BH) was announced by LIGO, a new era for astronomy began. Searches for electromagnetic (EM) counterparts of GW events is of fundamental importance, as they increase the confidence in the GW detection and help characterize the parameters of the merger. The Fermi gamma-ray space telescope has the best sensitivity to simultaneously observe a large fraction of the sky from $10 \mathrm{keV}$ to more than $300 \mathrm{GeV}$, providing the unique capability of rapidly covering the entire probability region from a LIGO candidate.

Here we present observations by the Fermi Gamma-Ray Burst Monitor (GBM) [1] and by the Large Area Telescope (LAT) [2] of the LIGO Gravitational Wave event GW150914, which has been associated to the merger of two stellar-mass BHs. We report the presence of a weak transient event in GBM data, close in time to the LIGO one. We discuss the characteristics of this GBM transient, which are consistent with a weak short GRB arriving at a large angle to the direction in which Fermi was pointing. Furthermore, we report LAT upper limits (ULs) for GW150914, and we present the strategy for follow-up observations of GW events with the LAT.
\end{abstract}

\section{GBM observations of GW150914}

On September $16^{\text {th }}, 2015$ the LIGO and Virgo collaborations reported that a candidate event had been identified in data recorded on September $14^{\text {th }}$ [3]. The candidate was subsequently characterized as being consistent with a signal from the merger of a stellar-mass BH binary system and was denominated GW150914 [4]. Although there are no predictions or well established mechanisms for detectable EM emission from these kind of mergers to guide a search for counterparts in the GBM data, we carried out a methodical search around the time and sky location of GW150914.

GBM has an instantaneous sky coverage of about $70 \%$, with the remainder blocked by the Earth. On September $14^{\text {th }} 2015$, GBM was recording data continuously from nearly $2 \mathrm{hr}$ before to over 7 hr after the GW event, without interruptions due to passages in the South Atlantic Anomaly (SAA).

\footnotetext{
ae-mail: elisabetta.bissaldi@ba.infn.it
} 


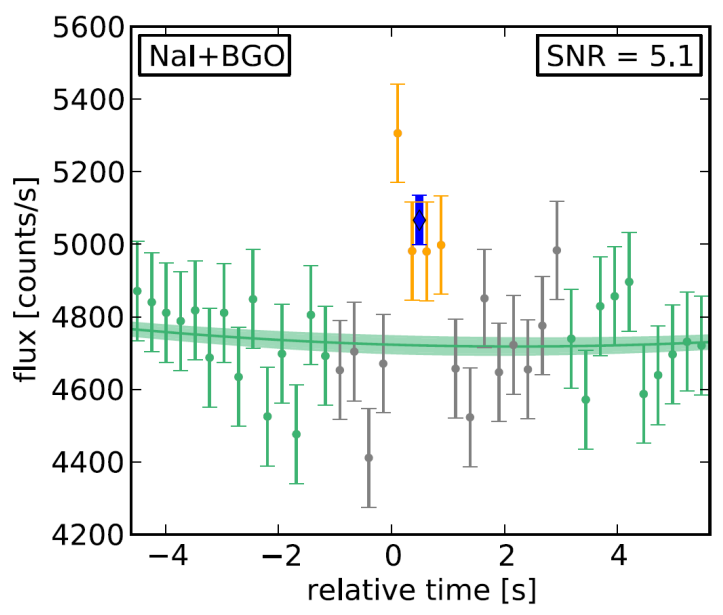

Figure 1. Model-dependent count rates detected as a function of time relative to the start of GW150914-GBM. The raw count rates are weighted and summed to maximize the signal to noise for a modeled source. Green data points are used in the background fit, gold points are the counts in the time period that shows significant emission, gray points are outside this time period, and the blue point shows an average over the gold points. For a single spectrum and sky location, detector counts for each energy channel are weighted according to the modeled rate and inverse noise variance due to background. The weighted counts from all NaI and BGO detectors are then summed to obtain a signal-to-noise optimized light curve for that model. A likelihood is assigned to each model by the targeted search based on the foreground counts (in the region of time spanned by the gold points), and this is used to marginalize the light curve over the unknown source location and spectrum.

GBM did not record any on board trigger around the time of the GW detection, at 09:50:45.391 UT. The triggers closest in time were from two events on the same day which are consistent with particle precipitation in or near the spacecraft, at 04:09:23 UT on entering the SAA and at 14:21:34 UT when Fermi was at high geomagnetic latitude, nearly $6 \mathrm{hr}$ before and $4.5 \mathrm{hr}$ after the GW event, respectively.

There are two offline search pipelines that check for impulsive events too weak to trigger on board Fermi, or from a sky position unfavorable to the two-detector on board triggering requirement. The first undirected search is based on analysis of the GBM Continuous Time-tagged Event (CTTE) data over four energy bands and 10 timescales, and it reported no candidates above the detection threshold on the day of the GW event. The second directed search is a targeted one [5]. By scanning both the GW and GBM data it aims to strengthen the significance of a sub-threshold signal in one data set by the detection of a signal in the other, provided that the false positive rate of the joint search is characterized and the detection levels in both instruments are selected accordingly. The directed search of the GBM data is seeded with the time and (optionally) the sky location of any LIGO/Virgo candidate event. A coherent search over all GBM detectors (NaI and BGO) using the full instrument response at each sky position is performed over a user-specified time window, assuming one of three template source spectra, revealing short-duration candidates typically between 0.256 to $8 \mathrm{~s}$ in duration. The candidates are ranked by a Bayesian likelihood statistic.

We searched $30 \mathrm{~s}$ of GBM data before and after the LIGO coalescence time for a plausible counterpart and found two candidate events. The first transient, occurring $11 \mathrm{~s}$ after GW150914, lasted $2 \mathrm{~s}$, was visible only below $50 \mathrm{keV}$ and its position was computed to be near the galactic center, well separated from and incompatible with the LIGO localization region. The second, hard transient occurred 
$0.4 \mathrm{~s}$ after GW150914, lasted $1 \mathrm{~s}$ and was reported in [6] as "GW150914-GBM". Figure 1 shows the model-dependent light curve of GW150914-GBM, where the detector data have been summed using weights that maximize the signal to noise for a given source model, and the unknown source model itself is weighted according to its likelihood in the data. Using $220 \mathrm{ks}$ of GBM data from September 2015, we calculated the FAR for GW150914-GBM to be $1.6 \times 10^{-4} \mathrm{~Hz}$. After considering both the frequency of occurrence and the proximity to the GW trigger time, we finally derived a post-trial false-alarm probability (FAP) of $0.0022(2.9 \sigma)$. In the following section we explore whether the GBM data for GW150914-GBM suggest an astrophysical origin and, if so, whether the source is consistent with GW150914 or can be attributed to other causes.

\subsection{Characteristics of GW150914-GBM}

Localization - GBM is capable of localizing an event from an arrival direction beneath the spacecraft, from which nearly equal count rates are expected in most of the NaI detectors. The rough localization of GW150914-GBM spans a region covering 3000 square degrees (68\% confidence level) and places the source direction underneath the spacecraft, at an angle of $163^{\circ}$ to the spacecraft pointing direction. This explains the unusual detector pattern of GW150914-GBM, with all of the individual detector count rates being slightly above background, simultaneously.

Spectrum - The data for GW150914-GBM imply a weak but significant hard X-ray source with a spectrum that extends into the $\mathrm{MeV}$ range. The count spectrum from the $\mathrm{NaI}$ detectors (summed) is consistent with the count spectrum from the BGO detectors (summed), indicating a reasonable physical spectrum that peaks in the BGO energy range. In order to compute the GBM instrument response functions we sample a range of 11 arrival directions along the observed LIGO location arc, using the data and associated responses for the detectors at each location that are most favorably oriented to the arrival direction. We find that for all considered directions, a power- law fit to the data from GW150914-GBM can be constrained. Weighting the sampling along the arc according to the LIGO localization probability contained near each point on the arc, we obtain a best-fit power-law index $-1.40_{-0.24}^{+0.18}$ and amplitude $0.002_{-0.001}^{+0.002}$ photons $\mathrm{s}^{-1} \mathrm{~cm}^{-2} \mathrm{keV}^{-1}$ yielding a fluence between 10 and 1000 $\mathrm{keV}$ of $2.4_{-1.0}^{+1.7} \times 10^{-7} \mathrm{erg} \mathrm{cm}^{-2}$. These are typical values for weak, short GRBs. If GW150914-GBM is part of the short GRB population, then its fluence is not atypical but its unfortunate arrival direction yields only a weak signal in GBM. At the time of the GW event, Fermi was at low geomagnetic latitude and was not near the SAA. While we cannot exclude a magnetospheric origin for GW150914GBM, the observing conditions were not conducive to such an event, nor is the light curve typical of magnetospheric activity, which is usually manifested as longer and smoother (tens of seconds) bumps above background. Moreover, using various search techniques, we found (i) no evidence for long-term steady emission from the direction of GW150914-GBM, (ii) no evidence for contamination by known sources of hard X-ray emission of any search for emission related to GW150914-GBM, and (iii) no evidence for non-impulsive emission related to the GW event in the days surrounding the event. A search for longer-term emission in the months before and after the GW event also revealed no signal.

\section{LAT observations of GW150914}

Fermi was operating in normal survey mode at the time of the LIGO trigger. Hence, the LAT autonomously observed the entire LIGO localization region within $\sim 70 \mathrm{~min}$ of the GW trigger, independently of any notification from LIGO, in the high-energy gamma-ray band. The LAT Collaboration reported a preliminary search throughout the LIGO localization area that did not reveal any new gamma-ray sources [7]. Here we describe LAT observations of the localization area of GW150914 
around the time of the trigger. We performed two complementary sets of searches for transient highenergy gamma-ray emission: automated searches that are performed routinely on all LAT data and targeted searches in the LIGO localization region on short and long time baselines that exploit the full sensitivity of the standard LAT analysis chain.

Automated searches to the counterpart for GW150914 were based on three main LAT pipelines: (i) The Burst Advocate (BA) Tool and the LAT Transient Factory (LTF); (ii) The Automated Science Processing (ASP); and (iii) the Fermi All-sky Variability Analysis (FAVA). None of the LAT pipelines found a possible counterpart to GW150914. During the $6 \mathrm{hr}$ interval containing the LIGO trigger GW150914, ASP detected $(>3 \sigma) 12$ known gamma-ray sources and 3 low-significance $(>1 \sigma)$ unidentified transients, none of which were consistent with the LIGO event localization.

For what concerns targeted searches, we checked different time windows by carrying out two customized analyses of the LAT data, which are based on the standard maximum likelihood analysis technique used for LAT data. In all of our searches we included in the likelihood model all sources (point-like and extended) from the LAT source catalog "3FGL" [8] as well as the Galactic and isotropic diffuse templates provided by the Fermi-LAT Collaboration ${ }^{1}$. We used the Pass 8 P8_TRANSIENTR010E_V6 event class and the corresponding instrument response functions.

\subsection{Short-baseline search}

This search focuses on the hours immediately after the GW trigger $t_{G W}$ and it is the most likely to find a counterpart to GW150914 if it is similar to a short GRB (sGRB). We consider a point in the sky observable by LAT if it is within the $65^{\circ}$ radius FoV and has an angle with respect to the local zenith smaller than $100^{\circ}$. The LAT coverage represents the integral of the probability densities of all points in the LIGO localization probability map observable by LAT at a given time. It was between $50 \%$ and $90 \%$ in the hours before the trigger, while at $t_{G W}$ the LAT was unfortunately viewing the opposite side of the sky from the LIGO localization region. Moreover, the coverage was zero until $t_{G W} \sim 4200$. The

${ }^{1}$ http://fermi.gsfc.nasa.gov/ssc/data/access/lat/BackgroundModels.html
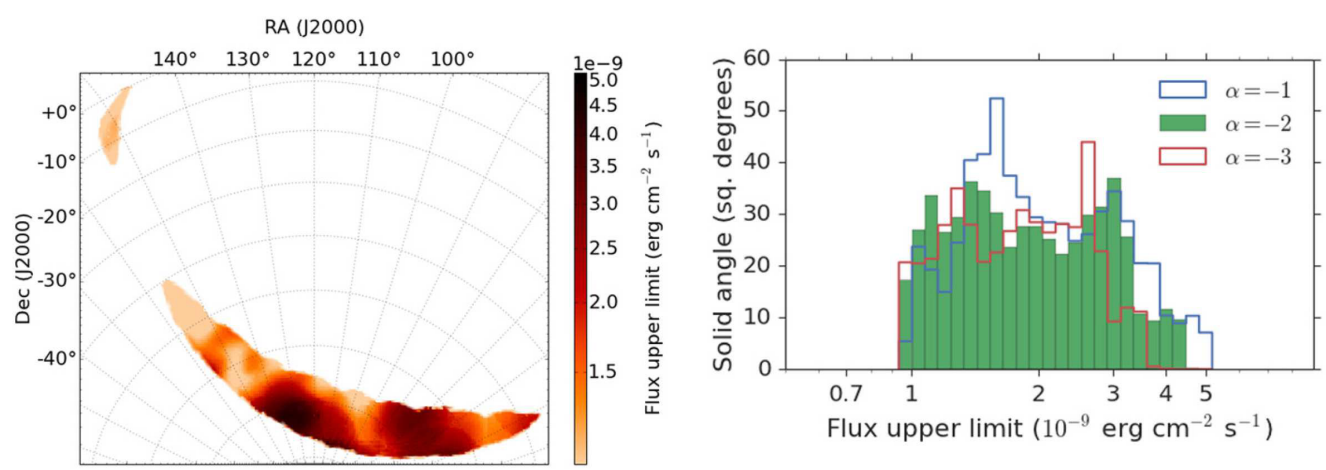

Figure 2. Flux upper limits ( $95 \%$ c.l.) in the energy range $100 \mathrm{MeV}-1 \mathrm{GeV}$ for $\mathrm{GW} 150914$ during the interval $T_{1}$ (4442-4867 s from $t_{G W}$ ). Left panel: the upper limits map covering the $90 \%$ region of the LIGO probability map. Right panel: the histogram of the upper limits in the map. We assumed a power-law spectrum for the source, with a photon index $\alpha$ of -2 (typical of afterglows of GRBs; green histogram), -1 (blue histogram), and -3 (red histogram). While the distributions are slightly different for the three cases, the ranges spanned by the upper limits are largely independent of the photon index. 
time interval $t_{G W}+4442-4867 \mathrm{~s}\left(T_{1}\right)$ had a coverage $>90 \%$, while during $t_{G W}+4867-10,000 \mathrm{~s}$ the coverage varied between $50 \%$ and $98 \%$, decreasing back to zero at around $t_{G W}+10 \mathrm{ks}$. We searched for a transient source in the time interval having more than $90 \%$ coverage $\left(T_{1}\right)$ and we did not find any significant excess.

We then derived upper limits for the gamma-ray flux of GW150914. Because the sensitivity of the LAT depends strongly on the angle from the source to the boresight of the instrument, the continuous variation of the LAT viewing direction in survey mode makes any flux limit for a particular source position time-dependent. Flux limits are also sensitive to astrophysical backgrounds, particularly in the Galactic plane, so that positions along the LIGO arc will have different flux limits even for the same observing conditions. These effects mean that flux limits vary according to both the time of observation and the position in the localization region. We show a map of the derived upper limits ( $95 \%$ c.1.) for the gamma-ray flux of GW150914 in the band $100 \mathrm{MeV}-1 \mathrm{GeV}$ in the left-hand panel of figure 2 and a histogram of the upper limits in the right-hand panel, both for interval $T_{1}$. Assuming a power-law spectrum for the source with a photon index of $\alpha=-2$, which is typical for GRB afterglows at LAT energies, the upper limits we find have a median of $1.7 \times 10^{-9} \mathrm{erg} \mathrm{cm}^{-2} \mathrm{~s}^{-1}$, and $5 \%$ and $95 \%$ percentiles of $0.9 \times 10^{-9}$ and $3.7 \times 10^{-9} \mathrm{erg} \mathrm{cm}^{-2} \mathrm{~s}^{-1}$, respectively. These upper limits are only weakly dependent on the choice of $\alpha$ as shown in the right-hand panel of figure 2 .

\subsection{Long-baseline search}

In this second search we considered data gathered during a two-month interval centered on $t_{G W}$. To increase the number of gamma-rays, we included all photons with energies between $60 \mathrm{MeV}$ and 100 $\mathrm{GeV}$. Because the PSF at $60 \mathrm{MeV}$ is broad, we applied a zenith cut of $95^{\circ}$ to further limit Earth limb contamination. We looked both for a long-duration signal of the order of one day as well as for a short-duration signal, but not necessarily in strict temporal coincidence with the LIGO trigger. To this end, we covered the entire $90 \%$ probability region provided by LIGO with a set of nine partially overlapping ROIs, each with a radius of $10^{\circ}$. For the first analysis of the second search, we divided the data in $10 \mathrm{ks}$ time bins. For each time bin and for each ROI, we calculated a TS map and determined the location of the grid position with the maximum $\mathrm{TS}\left(\mathrm{TS}_{\max }\right)$. We considered the position of $\mathrm{TS}_{\max }$ as the location of a possible counterpart and we ran an unbinned likelihood analysis adding a point source at the position of $\mathrm{TS}_{\max }$. This gave a value of $\mathrm{TS}_{\text {src }}$ (which is normally similar to $\mathrm{TS}_{\max }$ ). In these maps derived from low-statistics data, single high-energy gamma-rays can cause a high value of $\mathrm{TS}_{\max }$. To reduce the number of false positives from random coincidences, we required that the number of photons $N_{\gamma}$ that have a probability larger than 0.9 to be associated with the candidate counterpart to be greater than 2. No excesses met this requirement. We repeated the same analysis considering time bins of one day and again did not find any significant excess. We also considered the possibility of excesses over shorter timescales $(<1 \mathrm{hr})$, similar to the typical duration of high-energy emission from GRBs but not in temporal coincidence with the GW trigger. We calculated the entry and exit times for each ROI in the FoV of the LAT (a "FoV passage"), requiring that the distance between the LAT boresight and the center of the ROI be $<60^{\circ}$. In standard survey mode the duration of a FoV passage varies from a few hundred seconds to nearly one hour. Because we do not know if an EM signal would be in temporal coincidence with the GW signal, we searched for possible excesses in every passage, corresponding to a total of 6615 passages for each ROI. We did not detect any significant excess in any of the passages before or after $t_{G W}$ for any ROI.

To validate our interpretations of TS values we performed a detailed Monte Carlo simulation of two months of data (the same interval used in our analysis). The actual pointing history of the satellite was used; therefore, the correct exposure of the sky was automatically taken into account. All of the sources from the 3FGL catalog were kept fixed at their 3FGL catalog fluxes. As a result, the 
simulation is suitable for computing the distribution of TS under the null hypothesis that no transient signal is present. With the simulated data we repeated exactly the same analysis used on real data previously described. The Monte Carlo distributions proved to be a good match to the distributions of the $\mathrm{TS}_{\text {src }}$ values obtained from the flight data and the good absolute agreement is consistent with no statistically significant transient counterpart being present in the flight data. Also, given the large number of trials, relatively high values of TS can be obtained in Monte Carlo simulations even if no transient signal was added.

\section{Conclusions}

Future joint observations of GW events by LIGO/Virgo and Fermi-GBM could reveal whether the weak transient GW150914-GBM is a plausible counterpart to GW150914 or just a chance coincidence. As advanced LIGO begins operations, we eagerly anticipate the detection of gravitational waves in coincidence with a gamma-ray signal from GBM and LAT, likely from a short GRB arising from the merger of two neutron stars. Offline searches for weak GRBs that fail to trigger on-board Fermi indicate that additional short GRBs can be detected in the GBM data and dedicated analysis of LAT data can results in sub-threshold detections that can greatly improve our knowledge of the source of GW events and affect follow-up strategies for counterpart searches by other observers.

\section{Acknowledgements}

The Fermi-LAT Collaboration acknowledges support for LAT development, operation and data analysis from NASA and DOE (United States), CEA/Irfu and IN2P3/CNRS (France), ASI and INFN (Italy), MEXT, KEK, and JAXA (Japan), and the K. A. Wallenberg Foundation, the Swedish Research Council and the National Space Board (Sweden). Science analysis support in the operations phase from INAF (Italy) and CNES (France) is also gratefully acknowledged. E.B. is supported by the Italian Fondo di Sviluppo e Coesione 2007-2013 - APQ Ricerca Regione Puglia “Future In Research".

\section{References}

[1] V. Connaughton et al., The Astrophysical Journal Letters 826 L6 (2016)

[2] M. Ackermann et al., The Astrophysical Journal Letters 823 L2 (2016)

[3] The LIGO Scientific Collaboration \& Virgo, GCN 18330 (2015)

[4] B. P. Abbott et al., Physical Review Letters 116061102 (2016)

[5] L. Blackburn et al., The Astrophysical Journal Supplement Series 2178 (2015)

[6] L. Blackburn et al., GCN 18339 (2015)

[7] N. Omodei et al., GCN 18709 (2015)

[8] F. Acero et al., The Astrophysical Journal Supplement Series 21823 (2015) 